\title{
O uso de Coding Dojo no aprendizado colaborativo de programação de computadores.
}

\author{
Luiz Paulo Franz, UNIPAMPA, luizpaulofranz@gmail.com \\ João Pablo Silva da Silva, UNIPAMPA, joaosilva@unipampa.edu.br \\ Jean Felipe Patikowski Cheiran, UNIPAMPA, jeancheiran@unipampa.edu.br
}

\begin{abstract}
Resumo: Ensinar e aprender programação de computadores são atividades desafiadoras dos cursos vinculados à informática. Devido às dificuldades enfrentadas por estudantes, evidenciadas por altos índices de repetência e evasão, novas estratégias são necessárias para facilitar seu aprendizado e potencializar suas habilidades. Esse trabalho apresenta resultados parciais de um projeto desenvolvido na UNIPAMPA que é voltado ao apoio à aprendizagem e ao exercício de programação. O projeto propõe uma abordagem baseada em aprendizado colaborativo e na resolução de problemas, utilizando Coding Dojos como fio condutor. Foi observada participação ativa dos alunos dos cursos de informática da instituição e, embora a experiência tenha sido positiva para a maioria dos estudantes, o fator de exposição revelou-se um complicador a ser endereçado.
\end{abstract}

Palavras chave: Coding Dojo, aprendizado colaborativo, resolução de problemas, programação.

\section{Using Coding Dojo in collaborative learning of computer programming.}

Abstract: Teaching and learning computer programming are challenging activities related to computing courses. Due to the difficulties faced by students, evidenced by high retention and dropout rates, new approaches are needed to facilitate their learning and enhance their skills. This paper presents partial results of a project developed in the UNIPAMPA that is aimed to support learning and exercise programming. The project proposes an approach based on collaborative learning and problem solving, using Coding Dojos as base line. Active participation of students in courses related to computing was noticed and, although the experience has been positive for most students, the exposure factor proved to be a complicating factor to be addressed.

Keywords: Coding Dojo, collaborative learning, problem solving, programming.

\section{Introdução}

Historicamente, o aprendizado de programação de computadores é altamente desafiador para os estudantes de computação, dada a necessidade de raciocínio lógico-matemático imposto por essa habilidade, (WEBBER et al., 2010).

“O aprendizado de programação de computadores é um dos primeiros e maiores desafios encontrados pelos estudantes da área de computação e informática. As dificuldades encontradas pelos estudantes refletem-se em elevados índices de reprovação nas disciplinas relacionadas com o aprendizado das habilidades de programação e nas disciplinas que dependem diretamente dessas habilidades.” (ADÁN-COELLO et al., 2008, p. 10)

O curso de Engenharia de Software da UNIPAMPA tem em média uma retenção de 25\% nos componentes curriculares Algoritmos e Programação, Tipos Abstratos de Dados e Programação Orientada a Objetos. Esses dados são preocupantes e sugerem que as abordagens tradicionais de ensino nessa área (aulas expositivas e dialogadas, aulas em laboratório, exercícios extraclasse, trabalhos em grupo, etc.) nem sempre se mostram as mais eficientes. 
Atualmente, o ensino da programação no campus ocorre da seguinte forma: aulas em salas de aula, onde a fundamentação teórica é apresentada, e aulas em laboratório, onde a prática é realizada e alguns exercícios são propostos e resolvidos. Além disso, o campus conta com um ou mais monitores que são alunos anteriormente aprovados no componente curricular do qual são monitores e que ficam à disposição dos colegas para sanar dúvidas sobre o conteúdo em alguns horários da semana.

Contudo, os métodos de ensino e aprendizagem supracitados (comuns em instituições de ensino de informática e computação) parecem ser insuficientes devido à retenção e às dificuldades encontradas pelos discentes nessas disciplinas. Dessa forma, novas técnicas de aprendizagem devem ser introduzidas para atenuar problemas e oferecer mais espaços para os estudantes desenvolverem suas habilidades.

\section{Práticas de aprendizado colaborativo}

A literatura nos indica que práticas colaborativas auxiliam na consolidação do aprendizado nos indivíduos. Guzdial e Carroll (2002) afirmam que o diálogo sempre conduz ao conhecimento especialmente em ambientes colaborativos onde um conhecimento compartilhado é criado através do diálogo.

Essa forma de aprendizagem, conforme a teoria do equilíbrio de estruturas cognitivas de Piaget (1976), parte de uma situação inicial de desigualdade de habilidades e conhecimentos dos integrantes em um grupo e proporciona um espaço de interação e trabalho coletivo que leva os participantes a um reequilíbrio (ADÁN-COELLO et al., 2008). Esse reequilíbrio cognitivo, diferente do reequilíbrio de forças físicas, permite que o estado final possua melhor qualidade que o estado inicial para todos os envolvidos. O professor (ou tutor, ou instrutor) atua como mediador nesses exercícios, identificando obstáculos e fornecendo direções para resolução de dúvidas e conflitos que levem cada grupo ao aprendizado.

As práticas que estimulam o aprendizado colaborativo costumam ser diferentes nas universidades e na indústria de software. Destacam-se no ambiente acadêmico os trabalhos em grupo, as maratonas e os concursos de programação. Nas empresas, investe-se em práticas de programação em pares, capacitações e Coding Dojos.

\subsection{Na universidade}

O termo aprendizado colaborativo refere-se a uma forma de instrução na qual dois ou mais estudantes com diferentes níveis de conhecimento e habilidade trabalham com um objetivo comum. Assim, os próprios estudantes são responsáveis pelo aprendizado uns dos outros e de seu próprio (HE e HU, 2008). Além disso, esse tipo de prática exercita a habilidade de trabalhar em equipe, muito valorizada no mercado de trabalho desses futuros profissionais.

Trabalhos de programação em grupo são atividades comuns que estimulam organização, colaboração e autorresponsabilidade dos estudantes. Adan-Coello et al. (2008) salientam que são aspectos importantes na formação de grupos: evitar a constituição de agrupamentos homogêneos (alunos cujas habilidades estejam similarmente desenvolvidas) e evitar a constituição de agrupamentos gerados por afinidade (nos quais pode prevalecer acomodação ou sobrecarga de trabalho sobre participantes). Essa abordagem tradicional é eficaz, mas depende do acompanhamento do professor durante o trabalho e da capacidade de organização dos próprios alunos.

Maratonas e concursos de programação são atividades competitivas entre times que estimulam a resolução de problemas em um período específico de tempo. A Sociedade Brasileira de Computação (SBC) organiza anualmente, desde 1996, uma maratona de programação nacional com competições locais classificatórias que levam ao concurso 
de programação da Association for Computing Machinery (ACM) (SBC, 2014). Esse evento destina-se a alunos de graduação e pós-graduação nas diversas áreas da computação e fomenta nos alunos a capacidade de resolução de problemas, a criatividade e o trabalho em equipe.

\subsection{Na indústria de software}

De acordo com Lima et al. (2010), atualmente sabemos que o real valor das empresas são as pessoas que a compõe com seus talentos, seus conhecimentos e sua capacidade de disseminar esses últimos no ambiente de trabalho e aplicá-los de forma produtiva na organização. Nesse ambiente de constante competitividade e inovação, os métodos de aprendizado tradicionais se mostram insuficientes à demanda de aprendizado exigido nas organizações. Consequentemente, boa parte da capacitação do pessoal se dá experiencialmente, ocasionando um tempo gasto de forma não diretamente produtiva.

Nessa direção Melo et al. (2010) relatam que o aprendizado é produto da experiência e ele só ocorre ao tentar resolver algum problema. O aprendizado adquirido pela experiência aumenta o desempenho individual, dos times e da organização como um todo. Assim, o aumento do conhecimento técnico e o melhoramento das habilidades são processos contínuos de acúmulo de experiências.

A programação em pares é uma prática comum em métodos ágeis de desenvolvimento de software. Nascida na Programação Extrema (XP, do inglês), essa prática busca a constante revisão do código por dois programadores concomitantes, aumentando a produtividade e a qualidade do produto final pela redução de erros durante a programação e pela visão estratégia ampliada da dupla (BECK, 2004). O aprendizado colaborativo é uma consequência direta da codificação em pares praticada na indústria de software.

A prática de Coding Dojo realizada por algumas empresas de desenvolvimento de software permite que seja gerada uma situação de desequilíbrio amplo entre vários participantes. O grupo numeroso e heterogêneo de envolvidos (membros da equipe de desenvolvimento) deve trabalhar coletivamente na solução de um problema, compartilhando conhecimento e praticando a programação.

Os Coding Dojos são encontros organizados em torno de um ou mais desafios de programação nos quais os participantes são incentivados a participar e compartilhar suas habilidades de codificação com os demais para a resolução do problema (SATO et al., 2008). Como seus objetivos são o desenvolvimento de habilidades, a troca de experiências e a diversão, os desenvolvedores envolvidos os veem como um ambiente seguro para aprendizado e para testar novas ideias. Conforme Heinonen et al. (2013), o evento é organizado pelo chamado dojo master e dentre suas atribuições estão escolher os problemas a serem abordados e conduzir o evento. Como funcionamento básico, dentre os participantes do dojo, dois desenvolvedores são inicialmente escolhidos para resolverem um problema apresentado. Esses dois desenvolvedores trabalham juntos: um membro da dupla (piloto) assume o controle do computador - teclado e mouse enquanto o outro participante (copiloto) permanece auxiliando com ideias e indicando correções e melhorias. Os princípios de Desenvolvimento Guiado por Testes (BECK, 2011) conduzem o processo de desenvolvimento da solução. Os demais presentes participam como plateia, assistindo a codificação e as decisões tomadas pela dupla em direção à solução e discutindo entre si soluções alternativas e melhorias para a resolução do problema proposto. A plateia não interfere nas decisões da dupla ou opina, a menos que a dupla esteja travada e precise de ajuda. Depois de transcorrido um tempo préestipulado, há troca do piloto e do copiloto por participantes que estavam na plateia. 


\section{Proposta do projeto}

Visando o incentivo ao exercício da programação e as práticas colaborativas de aprendizado da academia e da indústria de software, um grupo de professores dos cursos de Ciência da Computação e Engenharia de Software da UNIPAMPA montaram uma proposta de criação de espaços extraclasse adicionais para troca de conhecimentos e experiências. Por meio do edital $n^{0} 140 / 2014$, a proposta descrita a seguir foi submetida e aprovada, permitindo a seleção de um discente bolsista que conduz os encontros sob orientação dos professores.

O projeto, entre outras atividades, propôs a realização de Coding Dojos como ambientes para aprendizagem e prática de programação de forma colaborativa. Para permitir o monitoramento do interesse e da participação dos alunos e a organização das atividades, uma disciplina no ambiente de aprendizagem Moodle foi criada com o nome Práticas

Colaborativas de Programação. Nela ficam centralizados todos os artefatos gerados ou usados durante o projeto (problemas propostos, programas construídos, enquetes realizadas, etc.) e o cronograma do projeto.

Antes dos eventos ocorrerem, um plano de divulgação iniciou-se, usando banners nos murais da universidade, envio de e-mails para listas de alunos e promoção em redes sociais. Apesar de o projeto ser mais direcionado aos cursos de informática da universidade, a divulgação se deu em todos os cursos, pois todos os cursos ofertados no campus Alegrete atualmente possuem a disciplina Algoritmos e Programação em sua matriz curricular.

Dentre as atividades realizadas no projeto, os Coding Dojos focados nas linguagens de programação C e Java (linguagens utilizadas nas disciplinas de programação dos cursos) foram escolhidos como objeto desse estudo.

\section{Metodologia de pesquisa}

Essa pesquisa concentrou-se em observar e registrar a participação de alunos de graduação de cursos de informática e engenharias em Coding Dojos. Adicionalmente, os participantes responderam questionários sobre sua experiência na dinâmica.

O bolsista selecionado para participar do projeto acompanhou todos os eventos de Coding Dojos e efetuou observação direta intensiva, assistemática e participante, conforme descrita por Marconi e Lakatos (2002). A participação dos estudantes foi registrada em todos os eventos e será apresentada nas próximas seções.

Adicionalmente, questionários com base nas recomendações de Marconi e Lakatos (2002) foram desenvolvidos na tecnologia Google Drive e disponibilizados aos participantes dos eventos.

O primeiro questionário buscou apenas identificar o perfil dos interessados e o segundo coletou dados sobre a experiência dos participantes do projeto. No segundo questionário, a maior parte das questões fundamentou-se na escala Likert (1932) e foi composta de afirmações a respeito da experiência nos Coding Dojos. A escala de concordância adotada determina a aceitação do sujeito em relação à afirmação imposta, variando de Discordo completamente a Concordo plenamente em cinco níveis e sendo de fácil interpretação pelos participantes. As últimas questões eram abertas e visavam identificar pontos positivos e negativos da prática. Os resultados preliminares da pesquisa são apresentados a seguir.

\section{Resultados}

O primeiro passo do projeto foi sondar através de um questionário divulgado pelos meios supracitados quais seriam os cursos com mais alunos interessados no aprendizado colaborativo de programação e o nível de interesse desses alunos. O resultado dessa 
investigação sugeriu que alunos dos cursos de informática (Ciência da Computação e Engenharia de Software) teriam maior representatividade e maior interesse, justificando nossas ações na instituição. O perfil dos 34 respondentes foi sumarizado nos gráficos da Figura 1.
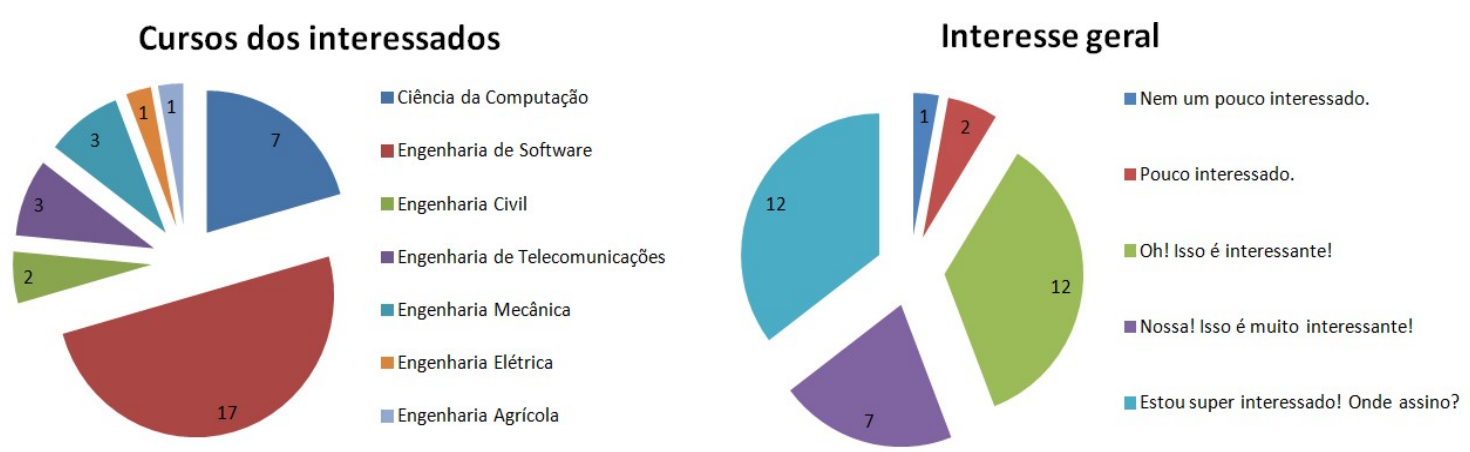

Figura 1 - Perfil dos alunos interessados que responderam ao formulário de sondagem.

Inicialmente, 52 alunos se inscreveram na disciplina de Práticas Colaborativas de Programação no ambiente Moodle, superando as expectativas da investigação preliminar. Dos inscritos, 30 estudantes participaram de, ao menos, um Coding Dojo. Uma visão geral da quantidade de participantes de cada evento e seus cursos é oferecida no gráfico da Figura 2.

\section{Participações por curso}

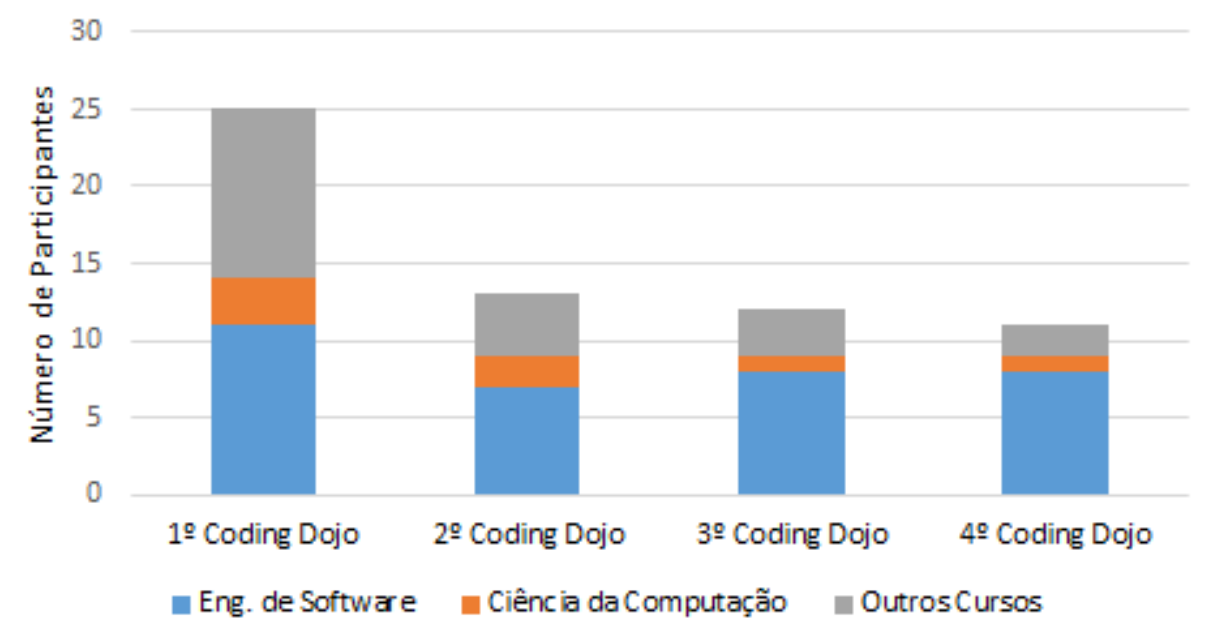

Figura 2 - Gráfico que sintetiza a participação nos eventos de Coding Dojo.

O primeiro Coding Dojo, realizado na linguagem de programação Java, contou com a presença de 25 pessoas. Essa grande quantidade de participantes se mostrou pouco produtiva, pois se observou que a prática dos Coding Dojos exige mais tempo de evento para grupos maiores. Dado que o tempo de programação de cada participante era de cinco a sete minutos, seriam necessárias mais de duas horas completamente dedicadas à programação para que todos os participantes assumissem papel de piloto e copiloto. Embora os encontros tenham duração de exatamente duas horas, grande parte do tempo (aproximadamente 30 minutos) são gastos na organização dos participantes, escolha dos problemas e encerramento da atividade. Dessa forma, com a grande quantidade de participantes, muitos saíram sem ter a oportunidade de codificar. 
Algumas adaptações tiveram que ser realizadas na estrutura padrão dos Coding Dojos. $\mathrm{O}$ alto número de pessoas, por exemplo, deixou alguns participantes inseguros e tímidos, e três alunos optaram por participar apenas como espectadores. Embora essa postura não faça tradicionalmente parte de Coding Dojos, seria invasivo obrigar a participação de todos e isso poderia afastar esses e outros interessados das próximas atividades. Adicionalmente, não foi utilizado o Desenvolvimento Guiado por Testes (BECK, 2011) como prática obrigatória, tendo em vista que o conhecimento prévio de testes unitários e de suas ferramentas dificultaria a participação de alunos de semestres iniciais dos cursos de informática e, principalmente, de outros cursos que não têm contato com a metodologia. Por fim, pausas periódicas foram realizadas para explicar o desenvolvimento de uma solução e garantir um melhor aprendizado. Geralmente, o dojo master (bolsista do projeto) guiava a explicação do raciocínio, mas os principais autores da solução participavam ativamente dos esclarecimentos.

O segundo evento de Coding Dojo, realizado na linguagem de programação C, teve uma redução drástica no número de participantes para 13, sendo a maioria dos desistentes externos aos cursos de informática. Vários alunos participaram apenas do primeiro Coding Dojo e possivelmente se afastaram do projeto ao perceberem que a dinâmica exigia alguns conhecimentos básicos sólidos de programação de computadores.

Na terceira edição, que foi realizada novamente na linguagem Java, o número total de participantes foi 12. Os participantes eram, praticamente, os mesmos do evento anterior. Na quarta e mais atual edição do evento até a submissão desse artigo, o Coding Dojo abordou novamente a linguagem $\mathrm{C}$ e teve um total de 11 participantes. Nesse encontro, por razões particulares, três participantes saíram antes do término da prática, reduzindo o grupo para apenas oito pessoas. Através da observação intensiva, constatou-se que o evento foi mais bem aproveitado com essa quantidade de participantes, pois as soluções foram mais dialogadas e as discussões envolveram todos os presentes. Foram apresentadas diferentes formas de abordar o problema e compor uma solução e, em alguns momentos, o quadro foi utilizado pelos participantes para explicar o raciocínio. Como identificado, o público alvo do projeto foi atingido, pois a maioria dos participantes vem dos cursos da área da informática. Os registros realizados também revelam que esse mesmo grupo foi mais assíduo e participativo que os demais.

\subsection{Percepções e depoimentos dos participantes}

Um questionário foi elaborado pelos pesquisadores para a coleta de dados para uma avaliação qualitativa das dinâmicas de Coding Dojo e obter depoimentos dos alunos. As informações obtidas dos 15 respondentes não possuem caráter estatístico, mas representam pontos de vista essenciais para identificarmos a eficácia da proposta e as fragilidades que podem ser contornadas durante sua execução.

Podemos observar na Figura 3 que a grande maioria dos participantes gostou e prefere trabalhar em equipe. Ainda, o aspecto colaborativo da dinâmica (ajudar e ser ajudado) também emergiu nas respostas.

Ainda na Figura 3, notamos que as questões de concentração, aprendizado e diversão não parecem consensuais. A despeito de seu aparente resultado positivo, esses tópicos demonstram que precisam de uma investigação mais profunda através de outras ferramentas.

Finalmente, é marcante que a grande maioria dos participantes se sentiu exposta durante seu momento de programação. Embora saibamos que essa exposição controlada no ambiente universitário é mais um exercício para o mercado de trabalho, dinâmicas que atenuem o constrangimento devem ser exploradas para evitar evasão dos participantes do projeto. 


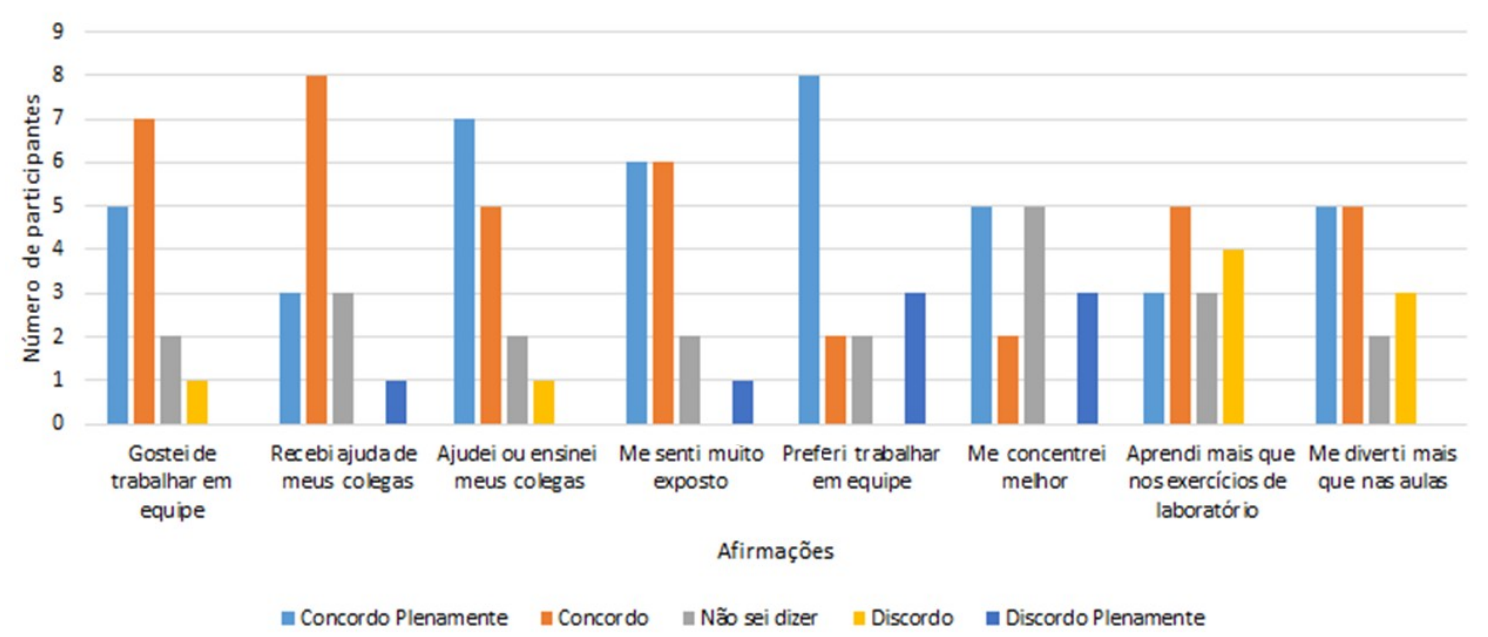

Figura 3 - Gráfico de satisfação dos participantes sobre os Coding Dojos.

Além dos dados apresentados acima, os depoimentos de alguns participantes ilustram os eventos. Eles ressaltaram pontos fortes e fracos da prática de Coding Dojos.

Depoimentos positivos:

"[o que mais gostei foi a] Interação geral e compartilhamento de conhecimentos”

"Aprendemos coisas novas [...]"

"Divertido"

"O que eu gostaria de frisar aqui, é que a dinâmica foi perfeita [...], no momento em que o piloto não sabia mais o que fazer todo mundo dava uma opinião, e daquela simples duvida se abria o ramo da conversa para outros pontos com direito a escrever no quadro, foi muito interessante, aprendi bastante [...]”

"Gostei muito da colaboração de todos os envolvidos para que os exercícios fossem resolvidos, sem que ninguém fosse tratado de forma diferente por não estar no mesmo nível de conhecimento.”

É importante também ressaltar que problemas foram identificados. O principal problema diz respeito à exposição causada pelo funcionamento da dinâmica.

\section{Depoimentos negativos:}

"sou tímida e fico com vergonha de ir na frente do pessoal, mas é algo comigo não com o coding dojo."

“[...] se expor na frente dos demais alunos, isto me gerou um extremo desconforto, pois me senti pressionada ao ponto de não conseguir desenvolver um raciocínio sobre o exercício que foi proposto, que me levou a sentir uma grande frustração.”

"Por não ter tanto conhecimento em relação a outras pessoas, e serem propostos exercícios que envolvam conhecimento mais aprofundado em programação, acho que me sinto despreparado em programar na frente das pessoas.”

Além disso, com os depoimentos foram recebidas algumas sugestões e críticas aos Coding Dojos realizados como, por exemplo, fazer mais eventos em uma única linguagem para fixar o conhecimento e adicionar um segundo copiloto (formando um trio de programadores em vez de uma dupla). 


\section{Considerações finais}

“A experiência de programar em grupo é muito interessante e divertida, além de ser agradável o método de ensinar e praticar a programação.” (participante do Coding Dojo).

No decorrer do projeto, com os registros realizados e com a análise dos dados coletados, podemos perceber que a ação foi, no geral, positiva e está cumprindo o objetivo de oferecer espaços de aprendizagem alternativos aos ambientes tradicionais. Embora a quantidade de participantes tenha sofrido reduções, observamos que os desistentes consistem principalmente de alunos de cursos fora da informática, justificando assim uma possível queda de interesse. Verificações mais detalhadas sobre a desistência de participação desses alunos serão realizadas e apresentadas em trabalhos futuros.

Os Coding Dojos mostram-se uma prática sustentável no desenvolvimento de habilidades de programação, embora sua dinâmica possa ser desconfortável para indivíduos com um perfil mais tímido ou inseguro, tendo em vista a exposição envolvida. Ainda estão sendo experimentadas maneiras de atenuar esse fator de exposição como, por exemplo, permitir que membros da plateia participem apenas com espectadores até que se sintam à vontade para programar com os colegas. Percebemos também que a dinâmica é mais produtiva e participativa ao utilizarmos grupos menores. Além do uso de grupos reduzidos permitirem a participação mais ativa de todos, reduzimos automaticamente a sensação de exposição. Foi observado que, quando o grupo esteve em seu menor número de participantes, os participantes com maior conhecimento e habilidade conversavam mais, discutindo as possíveis soluções para os problemas propostos, ao passo que os participantes com mais dificuldades em programação ou em compreender o problema e elaborar uma solução escutavam atentamente essa troca de ideias. Finalmente, pequenos ajustes tiveram que ser feitos a partir da dinâmica tradicional de Coding Dojos para tornar o evento mais flexível e atrativo aos alunos como, por exemplo, não utilizar Desenvolvimento Guiado por Testes durante o desenvolvimento e realizar pausas periódicas para explicar o raciocínio por trás de uma solução enquanto ela é desenvolvida.

Dentre as limitações dessa pesquisa, apontamos que o projeto ainda não está encerrado e que novos eventos de Coding Dojo ocorrerão. Assim, os dados dessa pesquisa podem ser ainda enriquecidos com comparativos periódicos e acompanhamento da mudança de opinião e postura dos participantes.

Como trabalhos futuros, destacamos a importância da investigação da mudança no desempenho dos alunos participantes dos Coding Dojos nas disciplinas relacionadas à programação. Temos como hipótese não testada que a participação nesses eventos contribuirá para um melhor desempenho dos alunos com histórico de dificuldades em disciplinas de programação. Essa teoria poderá ser verificada a partir do final do semestre letivo.

Por fim, consideramos que, em sua etapa atual, esse projeto já oferece contribuições significativas para apresentar Coding Dojos como uma prática de aprendizado colaborativo viável no exercício de habilidades de programação no ambiente universitário. 


\section{Referências}

ADÁN-COELLO, J. M.; MENEZES, W. S.; FARIA, E. S. J.; TOBAR, C. M. Conflito Sócio-Cognitivo e Estilos de Aprendizagem na Formação de Grupos para o Aprendizado Colaborativo de Programação de Computadores. Revista Brasileira de Informática na Educação, vol. 16, n. 03, 2008. p 9-19.

BECK, K. Programação Extrema Explicada: Acolha a Mudança. Porto Alegre: Artmed, 2004.

BECK, K. TDD: Desenvolvimento guiado por testes. Porto Alegre: Bookman, 2011. GUZDIAL, M.; CARROLL, K. Exploring the lack of dialogue in computer-supported collaborative learning. In: International Conference on Computer Supported Collaborative Learning, 2002, Boulder. Anais. Boulder: International Society of the Learning Sciences. p.418-424.

HE, X.; HU, W. An Innovative Web-Based Collaborative Learning Model and Application Structure. In: International Conference on Computer Science and Software Engineering, 5, 2008, Wuhan. Anais. Wuhan: Wuhan University/University of California, Irvine/University of Wisconsin La Crosse. p.56-59.

HEINONEN, Kenny et al. Learning agile software engineering practices using coding dojo. In: Proceedings of the 13th annual ACM SIGITE conference on Information technology education. ACM, 2013. p. 97-102.

LIKERT, R. A technique for the measurement of attitudes. Archives of Psychology. N. 140. New York: s.n, 1932. p. 1-55.

LIMA, E. B.; FERRAZ, S. F. S.; NETO, G. F. P.; LIMA, T. C. B. A aprendizagem experiencial em empresas de desenvolvimento de software. In: Congresso nacional de excelência em gestão, 6., 2010, Rio de Janeiro/Niterói. Anais. Niterói: Congresso nacional de excelência em gestão, 2010.

MARCONI, M. A.; LAKATOS, E. M. Técnicas de Pesquisa: planejamento e execução de pesquisas; amostragens e técnicas de pesquisa; elaboração, análise e interpretação de dados. $5^{\mathrm{a}}$ ed. São Paulo: Editora Atlas, 2002.

MELO, C. O.; SANTOS JR, C. D.; FERREIRA, G. R. M., KON, F. Um estudo exploratório dos fatores associados ao estímulo do aprendizado em times ágeis na indústria. In: Experimental Software Engineering Latin American Workshop, 7., 2010, Goiânia. Anais. Goiânia: Universidade Federal de Goiás/Instituto de Informática, p.80-98.

PIAGET, J. A equilibração das estruturas cognitivas. Rio de Janeiro: Zahar, 1976.

SATO, D. T.; CORBUCCI, H., \& Bravo, M. V. Coding dojo: An environment for learning and sharing agile practices. In: Agile 2008 Conference, 2008, Toronto. Anais. Toronto: Agile Alliance. p.459-464. IEEE.

SBC - Sociedade Brasileira de Computação. Maratona de Programação. Disponível em

$<$ http://www.sbc.org.br/index.php?option=com_content\&view=category\&layout=blog \&id=303\&Itemid=180>. Acesso em: 15 nov. 2014.

WEBBER, C., ZENATO, M., ARROSI, T. Caderno de Algoritmos: um Ambiente de Resolução de Problemas de Programação. Revista Brasileira de Informática na Educação, v. 8, n. 3, 2010. 